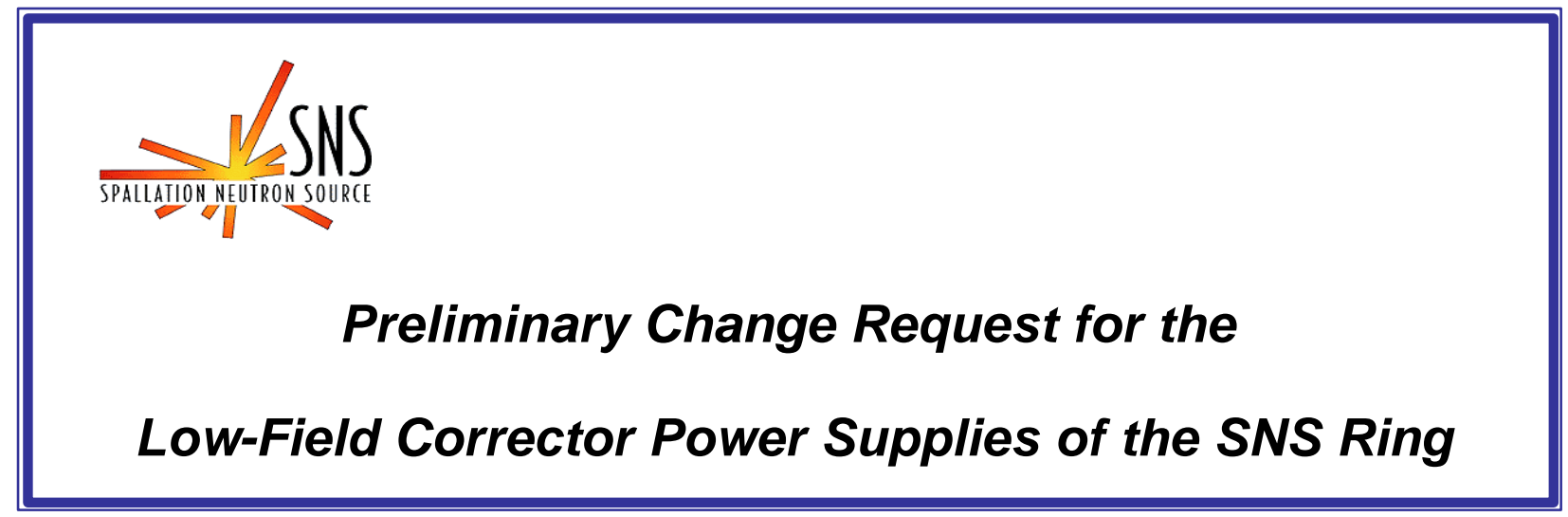

BNL/SNS TECHNICAL NOTE

NO. 089

I. Papaphilippou, C. Gardner, R. Lambiase,

Y.Y. Lee, I. Marneris and J. Wei

March 23, 2001

COLLIDER-ACCELERATOR DEPARTMENT

BROOKHAVEN NATIONAL LABORATORY

UPTON, NEW YORK 11973 


\title{
Preliminary Change Request for the Low-field Corrector Power Supplies of the SNS Ring
}

\author{
Y. Papaphilippou, C. Gardner, R. Lambiase, Y.Y. Lee, I. Marneris and J. Wei \\ Brookhaven National Laboratory, Upton NY, USA
}

March 21, 2001

\begin{abstract}
In view of the changes in the design for the accumulator ring of the SNS from the original FODO lattice [1] to the $220 \mathrm{~m}$ hybrid lattice [2] and finally $1.3 \mathrm{GeV}$ compatible $248 \mathrm{~m}$ ring [3], complementary studies have been undertaken, in order to review the powering of the low-field correctors of the SNS accumulator ring. In this note, we review the correction packages and present the accelerator physics studies for the adopted correction schemes and powering plan.
\end{abstract}

\section{The SNS accumulator ring lattice}

The latest design of SNS accumulator lattice is of a hybrid type consisting of four identical arcs and four special function straight sections. The arc has a FODO structure, with four cells (8 quadrupoles and dipoles) plus a quadrupole that matches the arcs with the straight sections. The latter contains two quadrupole doublets, setting the total number of quadrupoles at 52 . The magnetic elements are placed and powered in a way to preserve a four-fold symmetry. The space between the quadrupoles and dipoles is occupied by low-field correctors. They are adjacent to the quadrupoles (upstream or downstream) in order to take advantage of the horizontal or vertical beta function maximum in these locations of the ring [4]. A schematic presentation of one super-period is presented in Fig. 1.

\section{The SNS corrector packages}

A summary of the correction packages for the ring are described in Table 1. The correction winding elements either share a common magnet frame (dipole, skew quadrupole, skew sextupole), are wound on an main magnetic element (TRIM quadrupole) or stand alone as an individual magnet (dipole, sextupole, octupole). The power supply connection of each type of element is displayed graphically in Figs. 2 3. In the following paragraphs, we describe each system in more details. 


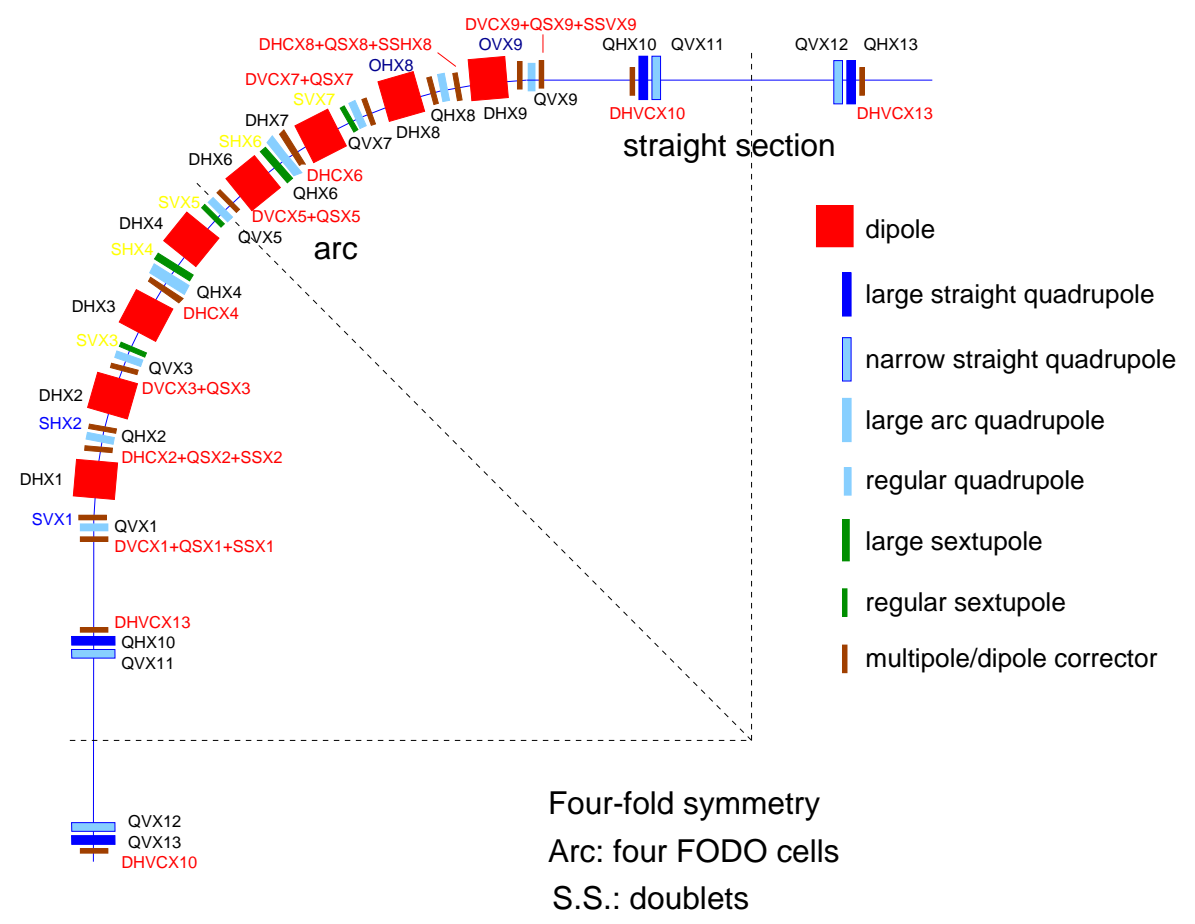

Figure 1: Schematic layout showing dipole, quadrupole, sextupole, and corrector magnets of one lattice super-period. The chromatic sextupoles shown in green are not in the baseline.

\subsection{Dipole correctors}

\subsubsection{System description}

The total number of dipole corrector magnets in the SNS ring is 44 . They are represented in Fig. 1 as brown rectangles. Due to the aperture change in the middle of the arc there are two types of dipole correctors in these areas: 28 of them have a $27 \mathrm{~cm}$ gap (27CDM30 in the magnet nomenclature corresponding to DVCX1, DHCX2, DVCX3, DVCX5, DVCX7, DHCX8 and DVCX9 of Fig. 1 and 8 are designed wider, with a 36cm gap (36CD30 corresponding to DHCX4 and DHCX6). The remaining 8 correctors (41CD30 or DHVCX10 and DHVCX13) are located in the straight sections, adjacent to the doublets (upstream and downstream) and their gap is even larger $(41 \mathrm{~cm})$.

Correctors in the arc produce either a horizontal or a vertical dipole kick and the determination of their positioning is imposed by the FODO structure: horizontal dipole correctors are placed near horizontal beta function maxima, adjacent to focusing quads and vertical dipole correctors are placed near vertical beta function maxima, adjacent to defocusing quads. On the other hand, the dipole correctors in the straight sections are designed as combined function magnets able to produce both horizontal and vertical dipole kicks. This will enable the necessary beam steering to any direction in the straight sections: in these areas, the beam envelope becomes wider and the closed orbit control is critical for injection, collimation, extraction and the RF system.

The dipole correction package allows the correction of closed orbit distortion during the 
Table 1: Summary of corrector elements for the Ring system

\begin{tabular}{|l|l|l|}
\hline Magnet & Mechanical Nomenclature & Number \\
\hline $\begin{array}{l}\text { Horizontal or Vertical Dipole + Skew } \\
\text { Quadrupole + Skew Sextupole (arc) }\end{array}$ & 27CDM30 & 28 \\
\hline Horizontal Dipole (arc wide aperture) & 36CD30 & 8 \\
\hline $\begin{array}{l}\text { Horizontal and Vertical Dipole (straight } \\
\text { section + linac dump) }\end{array}$ & 41 CD30 & $8+1$ \\
\hline Quadrupole TRIM Winding & 21QT40, 26QT38, 30QT58, 30QT44 & 52 \\
\hline Sextupole & 21CS26 & 8 \\
\hline Octupole & 21 CO26 & 8 \\
\hline Total number of elements & \multicolumn{1}{|c|}{121} \\
\hline Total number of functions & \multicolumn{2}{|c|}{} \\
\hline
\end{tabular}

accumulation period. This perturbation can be attributed to random quadrupole misalignments and dipole displacements (around $0.5 \mathrm{~mm}$ ), variation of the dipole field (of the order of a few $10^{-4}$ ), or random tilt of the dipoles (of about 0.5mrad) [5]. In order to achieve this, the dipole correctors are powered independently. The integrated dipole field of the arc correctors under design is around $6.7 \times 10^{-4} \mathrm{~T}$ m which gives the capability of 1.2 mrad kicks for each corrector, at $1 \mathrm{GeV}[6,7]$. The dipole correctors in the straight sections are able to produce approximately twice this integrated field and kick, both in the horizontal and vertical direction.

\subsubsection{Power supplies}

The total number of power supplies needed is 52, one for each arc dipole corrector and 2 for every combined function dipole corrector in the straight sections. The specified field can be given by running the power supplies at $11 \mathrm{~A}$. The baseline proposal for the power supplies procurement remain unchanged and consists of 5235 Volts power supplies able to deliver currents of $20 \mathrm{~A}$. By adding the 2 power supplies powering the 41CD30 dipole correctors in the ring injection dump, the total number becomes 54 .

\subsection{Quadrupole correction}

\subsubsection{System description}

The preservation of the lattice super-periodicity is essential for the good performance of highintensity rings. Based on first order perturbation theory, a lattice with perfect symmetry does not allow the excitation of resonances other than "structural", given by the condition: $k_{x} Q_{x}+k_{y} Q_{y}=m N$ where $m$ is the super-periodicity of the lattice (4 for the SNS ring) and $k_{x}, k_{y}$ and $N$ integers. On the other hand, when the super-periodicity is broken, e.g. by random errors in the magnets, "non-structural" resonances can be excited as well $(m$ can be any integer number). The combination of lattice perturbation in the presence of large space- 
charge forces in a high-intensity ring, can lead to excessive beam loss. This effect was already observed [9] and analyzed theoretically [11] in the KEK PS, where a 4th order "non-structure" space-charge induced resonance was excited, as the super-periodicity was broken due to errors in the quadrupole strengths.

The direct observable for a broken super-periodicity is the distortion of the linear optics functions. In the case of the SNS, random and systematic errors in the quadrupole strengths can create significant beta beating and dispersion distortion. Another perturbation comes from the injection chicane and bump which break the four-fold symmetry of the lattice and introduce additional beta wave [10]. In order to compensate these effects and allow a fine tuning of the SNS lattice, every magnet is equipped with TRIM windings able to produce $1 \%$ of the magnet quadrupole strength. The quadrupole gradient produced by the TRIMs is of the order of $5 \times 10^{-2} \mathrm{~T} / \mathrm{m}$, for $1 \mathrm{GeV}$ operation.

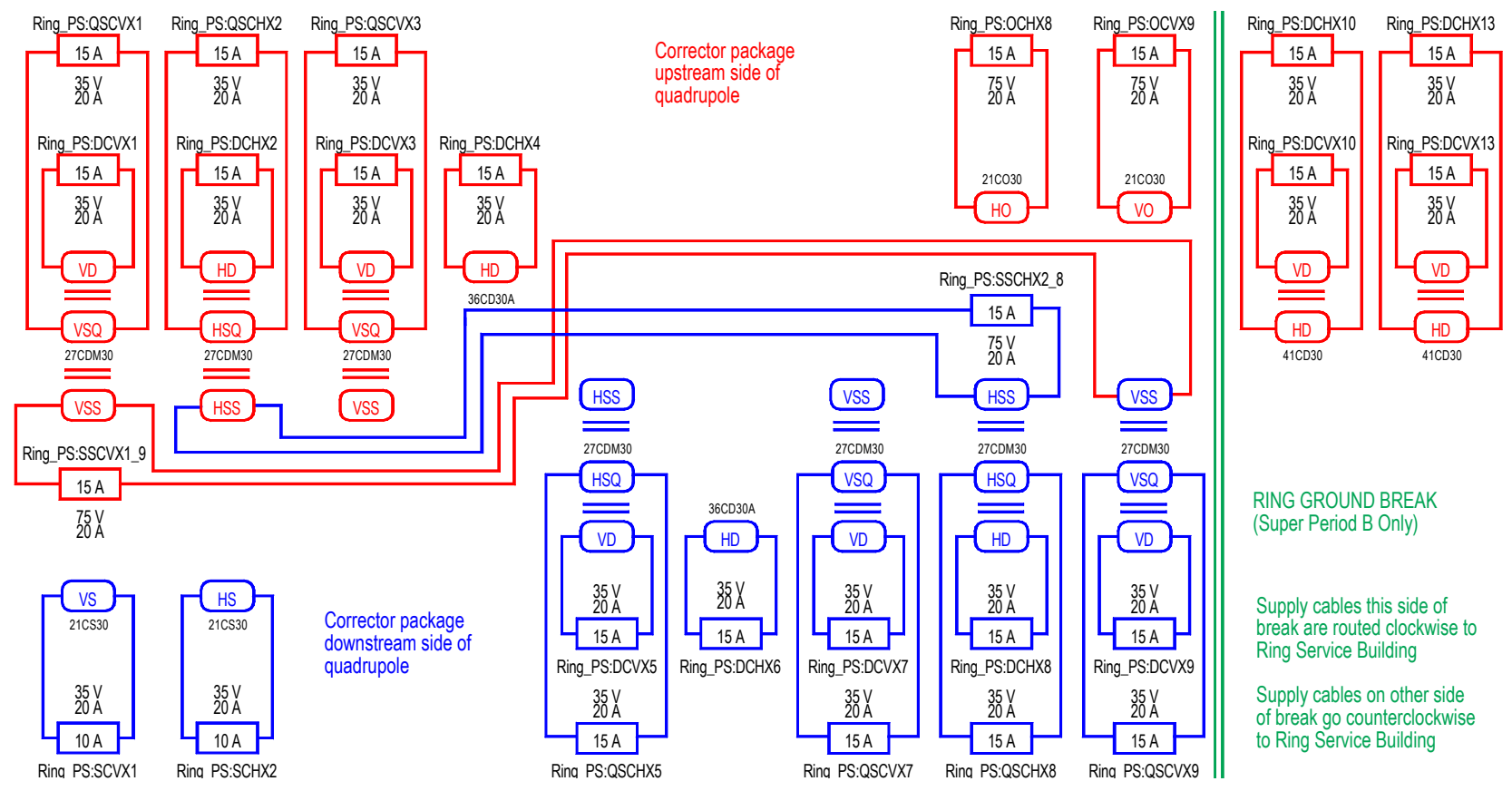

Figure 2: Schematic layout of the correctors' power supply connection for one super-period of the SNS ring.

Since the early stage of the project, correction schemes have been proposed for the linear optics distortions and quadrupole resonance compensation, by connecting appropriately in strings the quadrupole windings [8]. The total number of quadrupole string families proposed for the original FODO lattice was seven. With the evolution to the $228 \mathrm{~m}$ hybrid lattice and later to the $248 \mathrm{~m} 1.3 \mathrm{GeV}$ compatible ring, it became apparent that the number of families will be not enough for correcting all optics distortion. An individual powering scheme was proposed to give flexibility in the correction schemes, to assist the orbit correction in the early commissioning stage and to allow the beam-based alignment of the beam position monitors. A Preliminary Change Request (PCR) [12] was submitted requesting for individual powering 
of the quadrupole TRIM windings with 52 power supplies with an additional power supply for regulating the transfer function variation in the arc focusing quads which are powered in series but have different gap diameter due to the aperture increase in the middle of the arc. Due to the de-scoping of the SNS project, the original PCR was not approved. In this note, we propose a more restrictive correction scheme using 16 families of TRIM strings. Studies are under way in order to investigate this latest scheme for different working points and optics perturbations [13].

\subsubsection{Power supplies}

The total number of power supplies proposed is 16 . A preliminary connection scheme is shown in Fig. 3. In order to achieve the required $1 \%$ quadrupole gradient in each winding, for all five different types of quadrupoles, the power supplies should be able to give currents of 15 to $30 \mathrm{~A}$. The maximum power supply current and voltage delivered by the power supplies is $40 \mathrm{~A}$ and 35 Volts.

\subsection{Skew quadrupole correction}

\subsubsection{System description}

The skew quadrupole correctors are used for the linear decoupling of the horizontal and vertical motion of the beam. Coupling can be mainly attributed to random rolls in the quadrupoles of the SNS ring lattice, whose rms values can be as large as 1mrad. A smaller contribution comes from random and systematic skew quadrupole errors in the magnetic elements.

Apart from the linear optics perturbations such as beta and dispersion beating, skew quadrupole errors can excite the coupling resonances $Q_{x} \pm Q_{y}=N$. Especially for the nominal working point $\left(Q_{x}, Q_{y}\right)=(6.3,5.8)$ which was close to a structural coupling resonance $Q_{x}+$ $Q_{y}=12$, it was found essential that a very careful correction has to be applied in order to compensate the effect of random quadrupole rolls [14]. In a quite similar way as for quadrupole errors, the large space-charge tune-shift pushes the particles in the resonance and the result is a quite steep increase of beam loss to the $10 \%$ level. Moreover, it was shown that the global coupling correction would not be enough to cancel the effect and a local coupling scheme should be employed.

In the SNS ring there are no individual skew quadrupole magnets, but on the cores of the arc dipole correctors (27CDM30) separate windings are mounted that can create a skew quadrupole integrated gradient of about $2.25 \times 10^{-2} \mathrm{~T}$ at $10.9 \mathrm{~A}$. They are denoted as QSX1, QSX2, QSX3, QSX5, QSX7, QSX8, and QSX9 in Fig. 1. In the baseline scheme, it was foreseen to power only 16 of these windings, 4 per super-period, i.e. the ones at the beginning and the end of the arcs (QSX1, QSX2, QSX8, and QSX9). Due to the results mentioned above [14], it was apparent that this would not be sufficient. For this, we decided to power the skew quadrupole windings in every 27CDM30 correctors, which gives a total of 28 . A detailed coupling correction scheme is currently under study [15]. 


\subsubsection{Power supplies}

The total number of power supplies proposed is 28 . In order to achieve the required skew quadrupole gradient in each winding the power supplies should be able to give currents of $11 \mathrm{~A}$, for $1 \mathrm{GeV}$ operation. The maximum power supply current and voltage delivered is $20 \mathrm{~A}$ and 35 Volts.

\subsection{Skew sextupole correction}

\subsubsection{System description}

The excitation of skew sextupole resonances was found to be one of the most important limitations of the AGS booster. Skew sextupole resonances of the type $2 Q_{x} \pm Q_{y}=N$ and $3 Q_{y}=N$ can be excited by skew sextupole errors coming from rolls in the main dipoles or other geometrical errors in the magnet elements of the SNS ring. In order to correct this effect skew sextupole windings are mounted on the 27CDM30 dipole correctors along with the main dipole coils and the skew quadrupole components. The only one powered are the ones at the beginning an the end of the arc (SSX8 and SSX9). The integrated gradient is $6.6 \times 10^{-2} \mathrm{~T} / \mathrm{m}$ equivalent to the one of the correctors in the AGS booster.

In the initial scheme, the skew sextupole were powered in 2 families. This would have not been enough for correcting any skew sextupole resonance without changing the connection between correctors. Moreover it would necessitate power supplies producing higher voltage and power with an increased cost. Thus, it is required the additional powering of the skew sextupole windings in the 27CDM30 correctors at the beginning of the arc (SSX1 and SSX2) and the connection of the correctors in families of two: the first of the arc with the last and the second of the arc with the third are connected in series (the SSX1 with the SSX9 and the SSX2 with the SSX8, see Fig. 1), forming 8 families.

\subsubsection{Power supplies}

The total number of power supplies proposed is 8. In order to achieve the required skew sextupole field in each corrector, the power supplies should be able to give currents of $13 \mathrm{~A}$, for $1 \mathrm{GeV}$ operation. The maximum power supply current and voltage needed is $20 \mathrm{~A}$ and 35 Volts.

\subsection{Sextupole correction}

\subsubsection{System description}

The main functionality of the sextupole correctors is the correction of erect sextupole resonances of the type $3 Q_{x}=N$ and $Q_{x} \pm 2 Q_{y}=N$, which can be excited by sextupole errors in the dipoles, dipole fringe-fields at leading order and the small non-linear effect of the chromaticity sextupoles [16]. In the SNS ring, the sextupole correctors are 8 in total and are located at the beginning of the arc in non-dispersive areas in order to avoid "feed-downs" due to closed-orbit displacements giving a quadrupole effect at first order. The correctors 
are also taking advantage of the high horizontal or vertical beta function maximum in the respective locations. The names of these correctors in Fig. 1 are SVX1 and SHX1 and their mechanical name is $21 \mathrm{CS} 20$. The integrated sextupole gradient produced by the correctors is about $2.6 \mathrm{~T} / \mathrm{m}$.

For the same reason as for the skew sextupole correctors, it was decided to proceed with an individual powering scheme, with 8 power supplies instead of 2 as it was initially proposed. It will allow the possibility of correction of all resonant lines independently of the selected working point [16].

\subsubsection{Power supplies}

The total number of power supplies needed is 8. In order to achieve the required sextupole field in each corrector, the power supplies should be able to give currents of $8.4 \mathrm{~A}$, for $1 \mathrm{GeV}$ operation. The maximum power supply current and voltage delivered is $20 \mathrm{~A}$ and 35 Volts.

\subsection{Octupole correction}

\subsubsection{System description}

The octupole correctors are designated to compensate any octupole type of perturbation in the SNS ring. The main "octupole-like" contribution comes from the quadrupole fringe-fields to leading order, which are producing a tune-shift of the order of 0.025 at an amplitude of $480 \pi \mathrm{mm}$ mrad [17]. This is the third most important contribution in tune spread after space-charge and chromaticity.

The octupole correctors in the SNS ring are located at the end of the arc in non-dispersive areas in order to avoid sextupole "feed-downs" due to closed orbit displacements. They are 8 in total and as the other correctors, their position is determined by the maxima of the beta functions near the arc focusing and defocusing quadrupoles. The names of these correctors in Fig. 1 are OHX1 and OVX1 and their mechanical name is 21OC20.

In order to eliminate completely the large tune-shift coming from quadrupole fringe-fields, a third octupole family is necessary in areas where the horizontal and vertical beta functions are approximately equal, in the short straight sections, just next to the doublet $[17,18]$. Unfortunately, this is not possible to a first stage due to the tight space of the ring in these areas and budget issues.

On the other hand, it would be impossible to correct all octupole type resonances of the form $4 Q_{x}=N, 2 Q_{x} \pm 2 Q_{y}=N$ and $4 Q_{y}=N$ with the initial two-family scheme. For this reason, we proposed individual powering, in much the same way as for the other non-linear correctors. The integrated octupole gradient given by the correctors is $2.9 \mathrm{~T} / \mathrm{m}^{2}$, which will enhance the efficiency of resonance corrections.

\subsubsection{Power supplies}

The total number of power supplies needed is 8 . In order to achieve the required octupole field in each corrector, the power supplies should be able to give currents of $8.4 \mathrm{~A}$, for $1 \mathrm{GeV}$ operation. The maximum power supply current and voltage delivered is $20 \mathrm{~A}$ and 35 Volts. 
Table 2: Summary of low field power supplies for the ring system.

\begin{tabular}{|l|r|r|r|l|}
\hline Element & Baseline & PCR & Increase & Justification \\
\hline Dipole & 52 & 54 & 2 & Injection dump dipoles \\
\hline Quadrupole TRIM & 7 & 16 & 9 & $\begin{array}{l}\text { Large beta-beating due to lattice sym- } \\
\text { metry breaking (injection chicane/bump, } \\
\text { quadrupole transfer function, quadrupole } \\
\text { errors) }\end{array}$ \\
\hline Skew Quadrupole & 16 & 28 & 12 & Local tilt correction \\
\hline Skew Sextupole & 2 & 8 & 6 & $\begin{array}{l}\text { Skew sextupole resonance correction for } \\
\text { odd and even harmonics (AGS booster) }\end{array}$ \\
\hline Sextupole & 2 & 8 & 6 & $\begin{array}{l}\text { Sextupole resonance correction for odd } \\
\text { and even harmonics due to sextupole er- } \\
\text { rors and octupole feed-down }\end{array}$ \\
\hline Octupole & 2 & 8 & 6 & $\begin{array}{l}\text { Octupole resonance correction for odd } \\
\text { and even harmonics due to quadrupole } \\
\text { fringe fields }\end{array}$ \\
\hline Total & 81 & 122 & 41 & $\begin{array}{l}\text { Changes reflect the evolution of the de- } \\
\text { sign from the FODO lattice to the hybrid } \\
\text { and the latest 248m ring }\end{array}$ \\
\hline
\end{tabular}

These power supplies although increased in number need a factor of two less voltage then the initial ones.

\section{$3 \quad$ Benefits due to changes}

The SNS system has evolved considerably since the initial FODO lattice to the hybrid lattice of $228 \mathrm{~m}$ and finally to the $1.3 \mathrm{GeV}$ compatible $248 \mathrm{~m}$ ring. The powering of the correction system had to be reviewed following this evolution. In the initial baseline scheme, the total number of power supplies was 81 for the ring. In the present PCR, the total number of ring power supplies is 122 . The benefits of the increase for each correction scheme individually are stated in Table 2.

In general, the present powering capability enables us to apply the necessary correction schemes independently of the working point choice. This is instrumental for a high intensity machine such as the SNS ring with very stringent loss level requirements. The present number of power supplies reflects the absolute minimum needed, due to the budget restriction. It is most likely that this number will not provide the ability to achieve the high demands of the anticipated $1.3 \mathrm{GeV}$ operation of the SNS ring. In fact, it is debated that one may need more power supplies for independent control of the quadrupole TRIM windings and more skew quadrupole components for local decoupling schemes. We have also to stress that in the present package there are no spares which can be used if a power supply fails. In a first estimate on August 2000 and including all these supplementary power supplies in addition 
Table 3: Summary of cost for the low field power supplies for ring and transfer lines.

\begin{tabular}{|l|l|l|l|l|}
\hline Area & Number & Baseline Cost & PCR Proposed Cost & $\Delta$ Cost \\
\hline HEBT + Linac dump & $17+0$ & $\$ 436,203$ & $\$ 370,365$ & $-\$ 65,838$ \\
\hline Ring + Injection dump & $120+2$ & $\$ 1,669,724$ & $\$ 1,755,893$ & $\$ 86,169$ \\
\hline RTBT + Extraction dump & $14+2$ & $\$ 475,746$ & $\$ 405,156$ & $-\$ 70,590$ \\
\hline Cables & 157 & $\$ 1,907,800$ & $\$ 1,988,668$ & $\$ 80,868$ \\
\hline Total & & $\$ 4,48,473$ & $\$ 4,520,082$ & $\$ 30,609$ \\
\hline
\end{tabular}

to the individual powering of the transfer lines correctors [19] the total number came up to 250. This number was considerably reduced to the present number of 157 due to the project de-scoping (see Table 3).

On the other hand, the cost impact due to the present scheme is much smaller than the actual number increase of the power supplies. The original estimate per power supply was higher than the present one which is based on actual vendor quotes. In addition, due to the individual powering of most of the components the power supplies proposed have much smaller voltage and power requirements and less cables are needed. Finally, as most of the power supplies are of the same kind with maximum current and voltage specs of $20 \mathrm{~A}$ and 35 Volts, they can be possibly interchanged if necessary.

\section{Impact on cost and schedule}

The impact on the construction schedule is insignificant. The total number of power supplies including the ones for the transfer lines is 156 (see Table 3). The total unburdened cost increase from the old baseline is $\$ 30,609$. More details for each item can be found in Table 3 .

\section{Acknowledgments}

We would like to thank Viorel Badea, Jacques Negrin, Deepak Raparia, Nick Tsoupas, Joe Tuozollo and Wuzheng Meng for many usefull information. We would like also to thank Kerry Mirabella for her assistance in the cost estimates.

\section{References}

[1] W.T. Weng, et al., in the Proceedings of PAC'97, Vancouver, B.C., Canada, 1997; Y.Y. Lee, BNL/SNS Technical Note 26, 1997; C.J. Gardner, et al., in the Proceedings of PAC'97, Vancouver, B.C., Canada, 1997.

[2] J. Wei, et al., BNL/SNS Technical Note 66, 2000; J. Wei, et al., PRST-AB, 3:080101, 2000.

[3] J. Wei, et al., BNL/SNS Technical Note 76, 2000. 
[4] C.J. Gardner, BNL/SNS Technical Note 40, 1997.

[5] C.J. Gardner, BNL/SNS Technical Note 31, 1997; C.J. Gardner, BNL/SNS Technical Note 82, 2000.

[6] V. Badea and N. Tsoupas, Private communication.

[7] J. Negrin, SNS magnet parameter list (unpublished).

[8] C.J. Gardner, BNL/SNS Technical Note 37, 1997.

[9] S. Mashida and Y. Shoji, AIP Conf.Proc., 377, 160-168, 1995.

[10] J. Holmes, et al., to be presented in PAC'2001, Chicago, 2001.

[11] Y. Shoji and H. Sato, Nucl. Instr. Meth. Phys. Res., 399, 5-10, 1997.

[12] Y. Papaphilippou and N. Tsoupas (eds.), BNL/SNS Technical Note 75, 2000.

[13] Y. Papaphilippou, et al., BNL/SNS Technical Note, in preparation.

[14] A.V. Fedotov, et al., ICAP'00 Proceedings, Darmstadt, Germany, September 2000; A.V. Fedotov, et al., BNL/SNS Technical Note 86, 2001.

[15] N. Catalan-Lasheras, et al., BNL/SNS Technical Note, in preparation.

[16] N. Tsoupas, et al., EPAC 2000 Proceedings, Vienna, 2000.

[17] Y. Papaphilippou and D.T. Abell, EPAC 2000 Proceedings, Vienna, 2000.

[18] Y. Papaphilippou and D.T. Abell, BNL/SNS Technical Note 72, 2000.

[19] D. Raparia, private communication. 
Ring_Mag:QTHA2, 8 Ring_Mag:QTHC2, 8

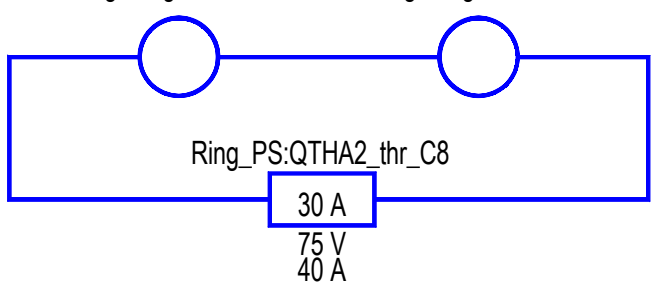

Ring_Mag:QTHA4, 6 Ring_Mag:QTHB4, 6

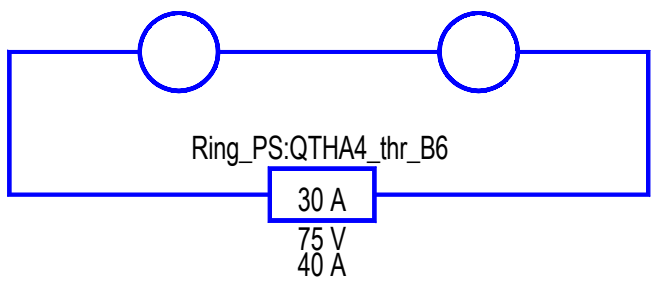

Ring_Mag:QTHA10, 13 Ring_Mag:QTHB10, 13

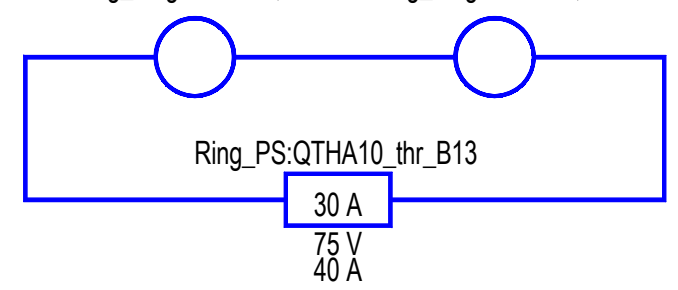

Ring_Mag:QTVA11,12 Ring_Mag:QTVC11, 12

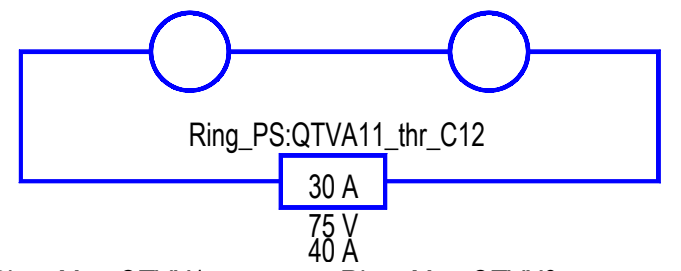

Ring_Mag:QTHB2, 8 Ring_Mag:QTHD2, 8

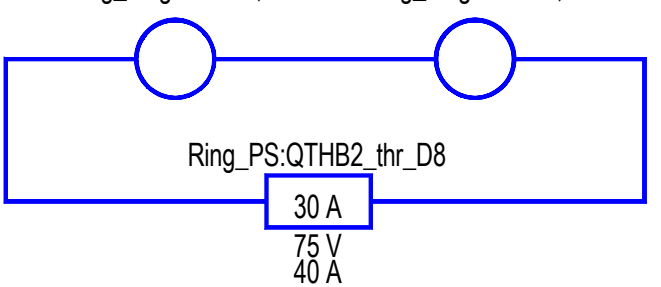

Ring_Mag:QTHC4, 6 Ring_Mag:QTHD4, 6

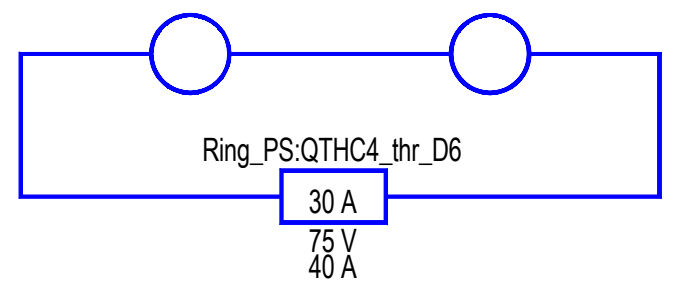

Ring_Mag:QTHC10, 13 Ring_Mag:QTHD10, 13

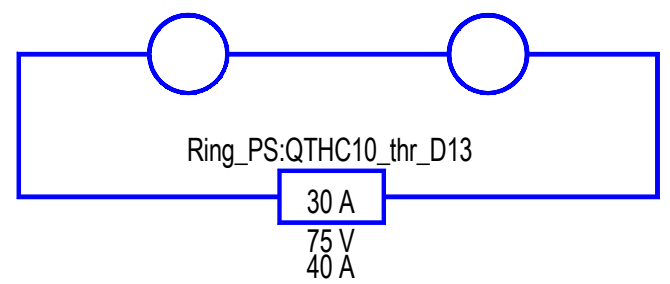

Ring_Mag:QVB11, $12 \quad$ Ring_Mag:QVD11, 12

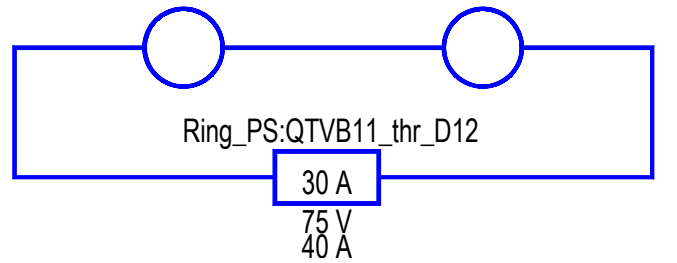

Ring_Mag:QTVX7

Ring_Mag:QTVX气

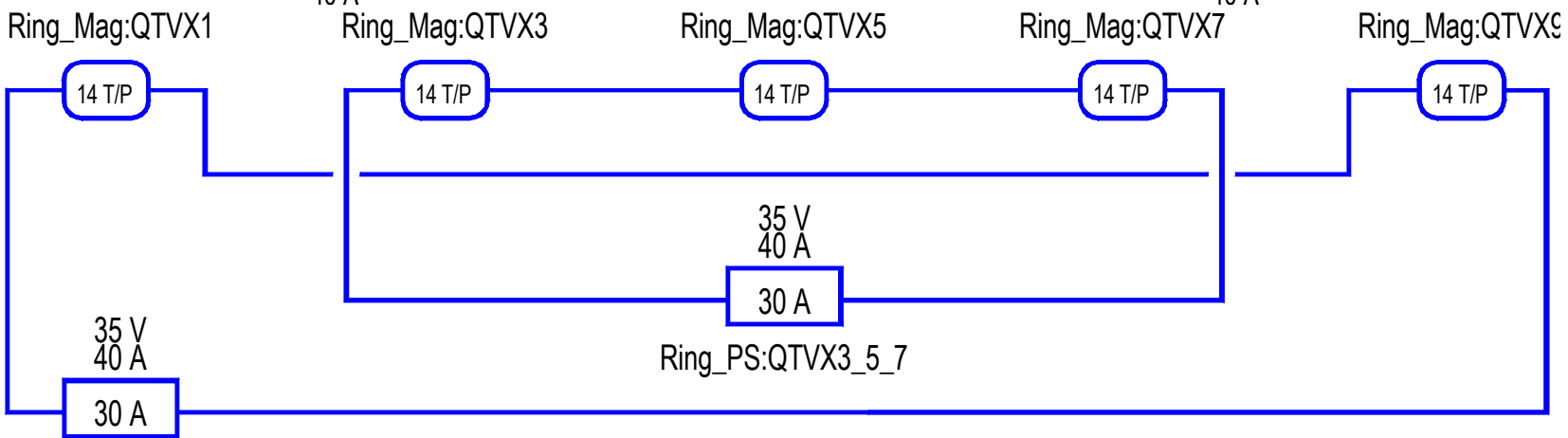

Ring_PS:QTVX1_9

Figure 3: Schematic layout of the TRIM strings' connection in the SNS ring. 